\title{
Vascular Endothelial Cadherin Modulates Renal Interstitial Fibrosis
}

\author{
Ikuyo Yamaguchia, b Bie Nga Tchao ${ }^{b}$ Megan L. Burger ${ }^{b}$ Muneharu Yamada ${ }^{b}$ \\ Toshitake Hyodo $^{b}$ Costanza Giampietro ${ }^{c}$ Allison A. Eddy ${ }^{a}$ b

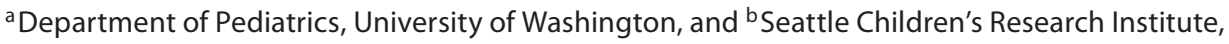 \\ Seattle, Wash., USA; 'The FIRC Institute of Molecular Oncology, Milan, Italy
}

\section{Key Words}

Vascular endothelial cadherin • Renal microcirculation •

Renal fibrosis • Unilateral ureteral obstruction

\begin{abstract}
Background/Aims: Renal interstitial fibrosis is a final common pathway of all chronic, progressive kidney diseases. Peritubular capillary rarefaction is strongly correlated with fibrosis. The adherens junction protein vascular endothelial cadherin (VE-cadherin) is thought to play a critical role in vascular integrity. We hypothesized that VE-cadherin modulates the renal microcirculation during fibrogenesis and ultimately affects renal fibrosis. Methods: Unilateral ureteral obstruction (UUO) was used as a renal fibrosis model in VEcadherin heterozygote (VE+/-) and wild-type (WT) mice, and the kidneys were harvested at days 3, 7, and 14. Peritubular capillary changes and fibrogenesis were investigated. $\boldsymbol{R e}$ sults: $\mathrm{VE}+/-$ mice had lower levels of VE-cadherin protein than WT mice at 3 and 7, but not 14 days after UUO. Vascular permeability was significantly greater in $\mathrm{VE}+/-$ mice 7 days after UUO, while peritubular capillary density was not significantly different in VE+/- and WT mice. Interstitial myofibroblast numbers and collagen I and III mRNA levels were significantly higher in VE+/- mice, consistent with a stronger early fibrogenic response. Expression of the pericyte marker
\end{abstract}

neuron-glial antigen 2 was upregulated after UUO, but was not greater in VE+/- mice compared to the WT mice. Conclusion: Our data suggest that VE-cadherin controls vascular permeability and limits fibrogenesis after UUO.

Copyright $\odot 2011$ S. Karger AG, Basel

\section{Introduction}

Chronic kidney disease (CKD) is a major health care problem that ultimately progresses to renal failure and the need for dialysis and/or renal transplantation [1]. While transplantation offers the best chance for longterm rehabilitation, most renal allografts are ultimately destroyed by chronic rejection [1-3]. In both native and transplanted kidneys, progressive fibrosis of the renal interstitium is the predominant final common pathway of renal destruction, regardless of the etiology of the original kidney disease [4-6].

During fibrogenesis, renal peritubular capillaries undergo major structural and functional changes, ultimately leading to capillary loss. Peritubular capillary rarefaction is correlated with the severity of fibrosis in rodent

B.N.T. and M.L.B. contributed equally to this paper.

\section{KARGER}

(c) 2011 S. Karger AG, Basel

Fax +41613061234

E-Mail karger@karger.ch

www.karger.com
Accessible online at: www.karger.com/nee
Ikuyo Yamaguchi, MD, PhD

Department of Pediatrics, University of Washington

Seattle Children's Research Institute, Center for Tissue and Cell Sciences, C9S-5

1900 Ninth Avenue, Seattle, WA 98101-1309 (USA)

Tel.+1 206884 7358, E-Mail ikuyo.yamaguchi@seattlechildrens.org 
CKD models such as 5/6 nephrectomy [7-9] and unilateral ureteral obstruction (UUO) $[10,11]$ and in human patients with chronic allograft rejection [12] and CKD $[13,14]$. The extent of capillary loss predicts interstitial damage and the decline in glomerular filtration rate in human subjects $[13,14]$. Capillary loss may propagate kidney damage due to hypoxia $[15,16]$ and/or decreasing nutrient supply to local regions.

Functional changes to capillaries also contribute to interstitial inflammation and subsequent fibrosis. Capillary endothelial cells promote inflammation by upregulating their expression of luminal adhesion molecules responsible for monocyte adhesion and transmigration [17]. Increased microvascular permeability may also contribute to interstitial inflammation by allowing chemoattractant plasma proteins such as oxidized albumin and fibrinogen to enter the interstitium. Enhanced permeability also increases fluid efflux, leading to tissue edema. Capillaries may also regulate fibrogenic responses via interactions with fibroblast precursors. Recently, Lin et al. [18] and Humphreys et al. [19] reported that pericytes and perivascular fibroblasts are a major source of renal interstitial myofibroblasts. As these cells are closely associated with the endothelium, it is likely that their differentiation is triggered in part by endothelial cell signals and/or endothelial cell death. In addition, evidence from lineage tracing studies performed by Zeisberg et al. [20] in mouse models of renal fibrosis suggests that endothelial cells may even transform into interstitial fibroblasts, a phenomenon called endothelial-to-mesenchymal transition.

An important molecular component of the capillary endothelial barrier that may regulate many of these structural and functional changes is the adherens junction protein vascular endothelial cadherin (VE-cadherin). VE-cadherin forms homophilic links with partners on the adjacent cell membrane [21] and is essential for vascular remodeling and the maintenance of vascular integrity [21-25]. Studies in cultured endothelial cells have shown that VE-cadherin limits the permeability of the endothelial monolayer [26]. In addition, VE-cadherin anchors a molecular complex that contains several important signaling molecules as well as cytoskeletal attachments. Thus, VE-cadherin can activate intracellular signals (e.g. kinase activation stimulated by VE growth factor) that are critical for endothelial cell proliferation and survival $[21,25]$. Based on these previous findings, we hypothesized that VE-cadherin has a critical role in the maintenance of capillary structure and function during CKD.

VE-Cadherin Modulates Renal Fibrosis
Because the homozygous deletion of VE-cadherin is embryonically lethal in mice, the present studies used VE-cadherin heterozygote (VE+/-) mice in the UUO model of CKD to investigate how the level of VE-cadherin expression affects peritubular capillary structure and function and the severity of renal interstitial fibrosis. Our results indicate that VE-cadherin was increased after UUO in wild-type (WT) mice; this upregulation was delayed in $\mathrm{VE}+/$ - mice, resulting in expression levels below WT on days 3-7 but similar to WT on day 14 . Studies comparing VE+/- and WT mice suggest that upregulation of VE-cadherin controls renal vascular permeability and may limit fibrosis.

\section{Animals and Methods}

\section{Animals}

The experiments were performed on WT and VE-cadherin heterozygote $(\mathrm{VE}+/-)$ littermate mice on a mixed Swiss background, which were generously provided by Dr. Elisabetta Dejana (The FIRC Institute of Molecular Oncology, Milan, Italy). The genotype was confirmed by polymerase chain reaction (PCR). VE-/- mice could not be used for these studies because the homozygous deletion is embryonically lethal.

\section{Renal Interstitial Fibrosis Model}

UUO or sham surgery was performed on 9- to 11-week-old male mice as described previously [27-29]. Kidney tissue was harvested 3,7 , or 14 days after UUO or sham surgery. Because previous studies showed no differences between these time points in sham-operated mice, a single time point (day 3 or 7) was used for sham controls in each experiment. All procedures were performed in accordance with the guidelines established by the $\mathrm{Na}$ tional Research Council Guide for the Care and Use of Laboratory Animals and were approved by the Institutional Animal Care and Use Committee at Seattle Children's Institute.

Histological Examination and Analysis

Immunohistochemical staining was performed on kidney tissues using procedures established in our laboratory $[27,28,30]$. Endothelial cells were stained using biotinylated or non-biotinylated rat anti-mouse CD31 (platelet endothelial cell adhesion molecule-1), monoclonal antibody (BD Pharmingen, San Diego, Calif., USA; 1:1,000 or 1:500). VE-cadherin staining was obtained using rat anti-mouse CD144 (BD Pharmingen; 1:100). The macrophage marker CD68 was stained using rat anti-mouse CD68 (Serotec Ltd., Oxford, UK; 1:100). The myofibroblast marker $\alpha$ smooth muscle actin ( $\alpha$-SMA) was stained using alkaline phosphatase-conjugated mouse anti-human $\alpha$-SMA 1A4 monoclonal antibody (Sigma-Aldrich, St. Louis, Mo., USA; 1:10). Pericytes were investigated by staining with rabbit anti-NG2 (anti-neuronglial antigen 2) chondroitin sulfate proteoglycan (Millipore, Billerica, Mass., USA; 1:500). The antibodies were visualized using the VECTASTAIN Elite ABC kit and VIP or Fast Red substrate (Sigma-Aldrich, St. Louis, Mo., USA). A Tyramide Signal Amplification Kit (Invitrogen, Carlsbad, Calif., USA) was used for im- 
munofluorescent staining of VE-cadherin, CD31, and NG2 according to the manufacturer's instructions. Briefly, cryosections were labeled with rat anti-mouse CD144, rat anti-mouse CD31, and/or rabbit anti-NG2 antibodies. The CD31 and CD144 fluorescence was amplified using horseradish peroxidase (HRP) conjugated anti-rat secondary antibody (Jackson ImmunoResearch) and Alexa Fluor ${ }^{\circledR} 488$-conjugated tyramide. NG2 was visualized using HRP-conjugated anti-rabbit and Alexa Fluor 594-conjugated tyramide. All sections were stained with DAPI or methyl green for nuclear visualization and mounted with VectaShield Fluorescence Mounting Medium or Vectamount (Vector Labs).

For analysis, the images $(\times 400)$ of 8-10 random, non-overlapping cortical fields per slide were captured using a SPOT digital camera (Diagnostic Instruction, Inc., Sterling Heights, Mich., USA). The CD68 or $\alpha$-SMA-stained tubulointerstitial area was quantified using Image-Pro Plus software (Media Cybernetics, Silver Spring, Md., USA) as described previously [27]. The results were expressed as the percentage of total tubulointerstitial area stained. An index of peritubular capillary density was determined by a grid method modified from previous studies [31, 32]. Each image (total area $=0.0625 \mathrm{~mm}^{2}$ ) was divided into $10 \times 10$ rectangular boxes (each $22 \times 28.5 \mu \mathrm{m}$ ), and boxes intersected by at least one CD31-positive peritubular capillary were counted. The maximal possible capillary staining index is 100 (i.e. every square in the grid contains at least one capillary), and the minimal index is 0 (no capillaries). Observers were blinded to the animal group at the time of analysis.

\section{Measurement of $m R N A$ Expression}

Total RNA was extracted from kidney tissue using TRIzol reagent (Invitrogen). The first-strand cDNA was generated by reverse transcription (iScript cDNA Synthesis kit, Bio-Rad Laboratories, Hercules, Calif., USA). Semiquantitative real-time PCR was performed with the iQ SYBR Green Supermix kit (Bio-Rad Laboratories) using the iCycler (Bio-Rad) standard protocol. The primers used for qRT-PCR were as follows: pro-collagen type I $\alpha_{1}$ forward 5'-CTTCACCTACAGCACCCTTGTG-3' and reverse 5'-GATGACTGTCTTGCCCCAAGTT-3', pro-collagen type III $\alpha_{1}$ forward 5'-TGAATGGTGGTTTTCAGTTCAG-3' and reverse 5'-GGTCACTTGCACTGGTTGATAA-3', fibronectin forward 5'-ACACGGTTTCCCATTACGCCAT-3' and reverse 5'-AATGACCACTGCCAAAGCCCAA-3', GAPDH 5'-AACTTTGTGAAGCTCATTTCCTGGTAT-3' and reverse 5' -CCTTGCTGGGCTGGGTGGT-3'. Standard curves were obtained for each gene. Reactions were run in triplicate, and the starting quantity of the mRNA of interest was measured and quantified relative to GAPDH.

\section{Immunoblotting}

Pieces of frozen kidney were homogenized in RIPA buffer supplemented with PMSF, protease inhibitor cocktail, sodium orthovanadate, and NaF (Santa Cruz Biotechnology, Inc.). The protein concentration was determined using the BCA protein assay (Pierce Biotechnology, Rockford, Ill., USA). Protein samples (20 $\mu \mathrm{g})$ were separated by electrophoresis in $4-15 \%$ pre-cast polyacrylamide gels (Bio-Rad Laboratories, Hercules, Calif., USA) and transferred to nitrocellulose membranes. The primary antibodies used were goat anti-mouse VE-cadherin (R\&D Systems, Inc.; $1: 2,000), \beta$-actin $(1: 2,000)$, monoclonal anti- $\alpha$-SMA (clone 1A4; Sigma-Aldrich; 1:2,500), and rabbit anti-NG2 chondroitin sulfate proteoglycan (Millipore; 1:1,000). The secondary antibodies were IRDye ${ }^{\mathrm{TM}} 800$ infrared dye-labeled antibodies (Rockland, Gilbertsville, Pa., USA), and Alexa Fluor 680-labeled antibodies (Molecular Probes, Eugene, Oreg., USA). Protein bands were visualized using the Odyssey Infrared Imaging System (LI-COR Biosciences, Lincoln, Nebr., USA) for fluorescent Western blots. All Western blot results were normalized by dividing each fluorescence measurement by that of $\beta$-actin in the same sample. Lanes in some representative blots shown in the figures have been rearranged for correspondence with the text; vertical lines indicate where data from different blots or non-adjacent lanes have been juxtaposed.

Qualitative Evaluation of Vascular Permeability by Injection of Fluorescein-Conjugated Dextran

Fluorescein-conjugated dextran (FITC-dextran; 500 kDa, anionic, lysine fixable) (Invitrogen) was dissolved in normal saline at $7.5 \mathrm{mg} / \mathrm{ml}$, and $150 \mu \mathrm{l}$ was injected into the tail vein [33]. Kidneys were harvested after $10 \mathrm{~min}$ of incubation and fixed in $10 \%$ formalin overnight. Sections of paraffin-embedded tissue were examined, and images were captured using a fluorescence microscope equipped with a SPOT camera.

Measurement of Vascular Permeability using Evans Blue Dye

Vascular permeability was determined by intravascular injection of Evans blue dye (Sigma-Aldrich). Evans blue $(20 \mu \mathrm{g} / \mathrm{g}$ mouse body weight, $2 \mathrm{mg} / \mathrm{ml}$ in $0.9 \% \mathrm{NaCl}$ ) was injected into the mouse tail vein. After $30 \mathrm{~min}$, dye remaining in the vessels was washed out by perfusing the mouse with $0.9 \% \mathrm{NaCl}$ via a needle inserted into the left ventricle of the heart. The mouse was then euthanized immediately, and the kidneys were removed. Each kidney was cut into two pieces along the horizontal plane, and both pieces were weighed. One piece was used for Evans blue extraction with $100 \%$ formamide ( $4 \mathrm{ml} / \mathrm{g}$ wet kidney weight) at room temperature for $24 \mathrm{~h}$. The other piece was dried for $24 \mathrm{~h}$ at $55^{\circ} \mathrm{C}$ and weighed in order to obtain the ratio of dry weight to wet weight. The extracted dye was measured by spectrophotometry (absorbance at $620 \mathrm{~nm}$ ) using a 96-well plate reader. The results were calculated from a standard curve of Evans blue dye (0.05-50 $\mu \mathrm{g} / \mathrm{ml})$ and expressed as $\mu \mathrm{g} / \mathrm{g}$ dry kidney weight $[34,35]$.

Data Analysis and Statistics

Data are expressed as mean \pm standard deviation unless indicated otherwise in the figure legends. Statistical significance was evaluated by $t$ test and one-way or two-way ANOVA using StatView version 5.0 software. $p$ values $<0.05$ were considered statistically significant.

\section{Results}

\section{Changes in VE-Cadherin Expression after UUO}

To investigate the role of VE-cadherin in the UUO model, VE-cadherin mRNA and protein expression were measured in the WT mouse kidneys after sham and UUO surgery. qRT-PCR and Western blotting showed that VEcadherin mRNA and protein were significantly elevated 

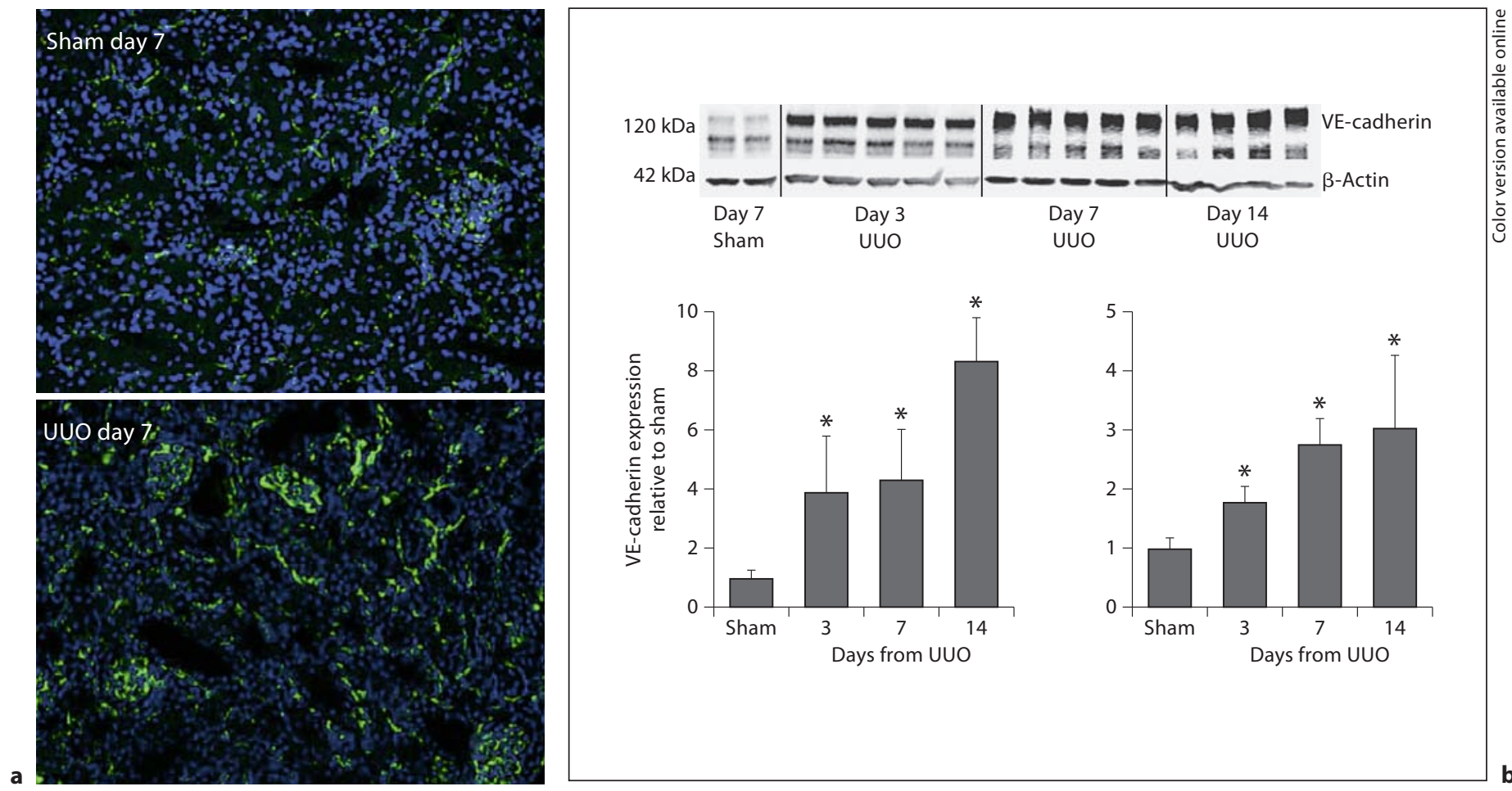

Fig. 1. VE-cadherin expression after UUO in the WT mouse. a Immunostaining for VE-cadherin in frozen kidney sections prepared 7 days after sham surgery (top) or UUO (bottom). $\times 200$. b Top, representative Western blot showing VE-cadherin $(\sim 120$ $\mathrm{kDa})$, shed VE-cadherin $(\sim 100-110 \mathrm{kDa})$, and $\beta$-actin $(42 \mathrm{kDa})$. Bottom right, VE-cadherin protein (120-kDa band) measured by
Western blotting, normalized by $\beta$-actin. All values are expressed relative to the mean sham level. ${ }^{*} \mathrm{p}<0.05(\mathrm{n}=5-7)$. Bottom left, relative expression of VE-cadherin mRNA 3, 7, and 14 days after sham surgery and UUO, measured by qRT-PCR and normalized by GAPDH. All values are expressed relative to the mean sham. ${ }^{*} \mathrm{p}<0.05(\mathrm{n}=4-6)$.
Fig. 2. Relative VE-cadherin expression in $\mathrm{VE}+/$ - and WT mice. Top, representative Western blot. Bottom, VE-cadherin protein expression. VE+/- mice showed lower VE-cadherin protein expression at days 3 and 7 but not at day 14 after UUO. All values are expressed relative to the mean WT sham. ${ }^{*} \mathrm{p}<0.05(\mathrm{n}=5-7)$.

VE-Cadherin Modulates Renal Fibrosis

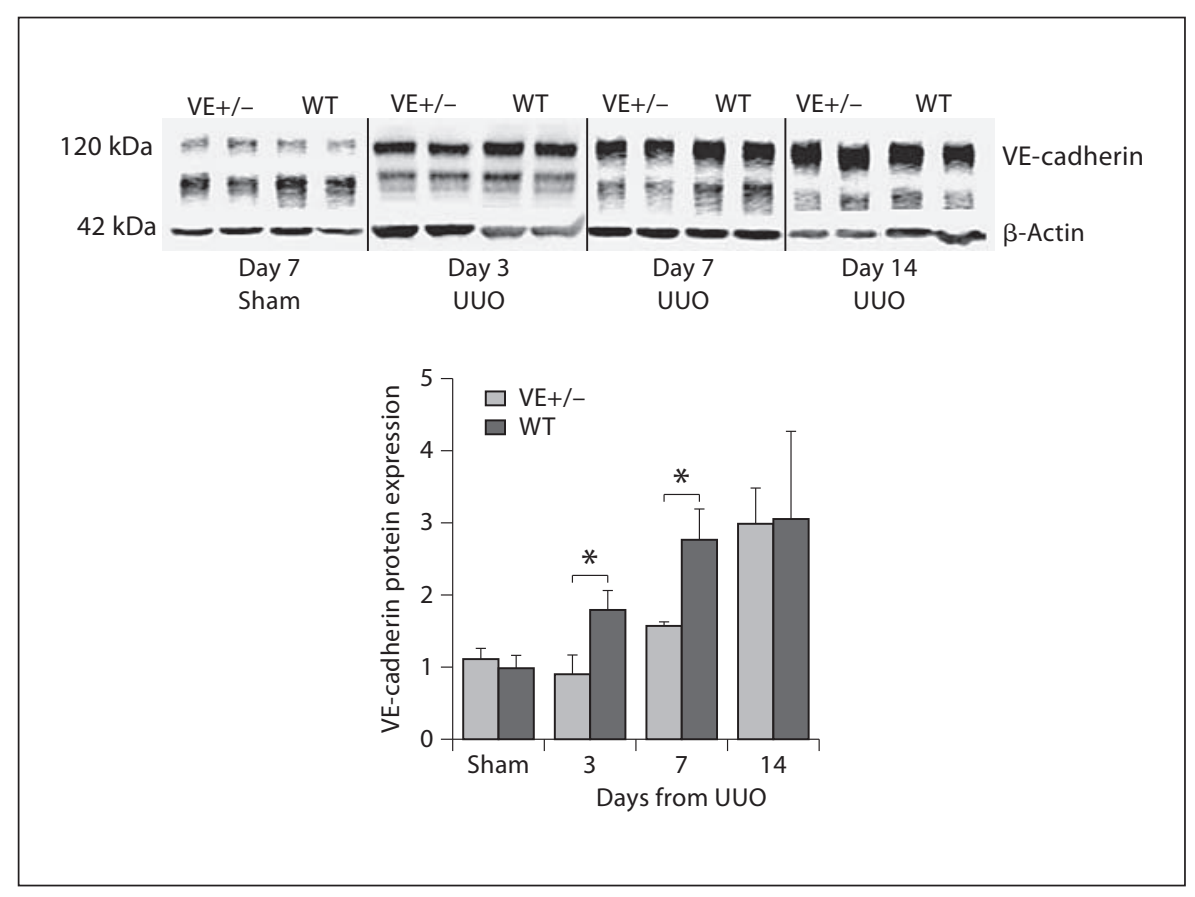

Nephron Exp Nephrol 2012;120:e20-e31 
3, 7 and 14 days after UUO (fig. 1). Immunofluorescent staining confirmed stronger staining 7 days after UUO (fig. 1). Thus, any loss of vascular integrity in the UUO model of CKD (see results below) cannot be explained by a decrease in total VE-cadherin protein. Instead, the observed increase in VE-cadherin may represent a compensatory mechanism that limits vascular damage and/or hyperpermeability (see below).

\section{VE-Cadherin Expression in Heterozygote Mice}

To investigate whether the level of VE-cadherin expression modulates interstitial fibrosis, we first determined whether deletion of one copy of the VE-cadherin gene affects the level of VE-cadherin protein. After sham surgery, VE-cadherin levels did not differ significantly between $\mathrm{WT}$ and $\mathrm{VE}+$ /- mice. After UUO, VE-cadherin expression increased in both $\mathrm{WT}$ and $\mathrm{VE}+/-$ mice. How ever, $\mathrm{VE}+/-$ mice showed a delayed response, with significantly lower expression at days 3 and 7 (fig. 2). VEcadherin expression at day 14 did not differ between WT and $\mathrm{VE}+/-$ mice. Thus, the $\mathrm{VE}+/-$ mice provided a good system to investigate the early effects of the VE-cadherin expression level during chronic renal injury days 3-7 after UUO, but were not well suited for investigating the effects of VE-cadherin at later time points.

\section{Peritubular Capillary Loss}

Peritubular capillaries were indentified by CD31 immunofluorescent staining of frozen kidney sections from WT mice. In sham-operated mice, a large number of peritubular capillary cross sections were positive. After UUO, the density of positive capillaries decreased progressively (fig. 3a). Although VE+/- mice showed a trend toward lower peritubular capillary density at day 7 , the differences were not statistically significant between $\mathrm{VE}+$ /- and WT mice after sham or UUO (fig. 3b).

\section{Renal Vascular Permeability}

As an initial method to assess the changes in vascular permeability after UUO, a qualitative visualization using dextran $(500 \mathrm{kDa})$ conjugated to fluorescein isothiocyanate (FITC) was employed. Thirty minutes after intravenous injection of FITC-dextran, the fluorescent label was detected within the interstitium in UUO kidneys (day 7), whereas labeled dextran appeared largely confined to intravascular areas in sham-operated kidneys (fig. 4a). These results suggest that capillary permeability is increased in response to UUO, allowing large macromolecules to enter the interstitium. However, the presence of the intravascular fluorescent label and the autofluorescence of kidney tissue limited our ability to quantify the change using this method. Therefore, we quantified the permeability in WT and VE+/- mice 7 days after UUO using a different method based on intravascular injection of Evans Blue dye. After $30 \mathrm{~min}$ following the injection, dye remaining in the vessels was washed out by perfusing the mouse with $0.9 \% \mathrm{NaCl}$ before harvesting the kidney. On visual inspection, the obstructed left kidney showed strong staining, whereas the contralateral kidney retained much less dye (fig. $4 \mathrm{~b}$ ). The measured tissue Evans Blue levels were greater in $\mathrm{VE}+/-$ mice than in WT mice (fig. 4c). These data support the hypothesis that rapid upregulation of VE-cadherin expression in WT mice limits the increase in renal vascular permeability after UUO.

\section{Macrophage Accumulation}

Interstitial inflammation during CKD is thought to depend in large part on the transmigration of circulating monocytes and their differentiation into macrophages $[4,36]$. Therefore, we investigated whether $\mathrm{VE}+/-$ and WT mice show a difference in interstitial macrophage accumulation after UUO. CD68+ interstitial macrophages were present in the interstitium after UUO, whereas few cells were detected after sham surgery. The percentage of tubulointerstitial area with positive CD68 staining increased rapidly and peaked at day 7 after UUO. VE+/- and WT mice showed no difference in CD68+ macrophage infiltration in the interstitium (fig. 5). An additional study using F4/80 immunostaining confirmed that there was no difference in interstitial macrophage density between VE+/- and WT mice (data not shown). These data suggest that differences in the fibrogenic response between VE+/- and WT mice (see below) do not result from an alteration of total macrophage accumulation.

\section{Activated Fibroblasts}

Activated fibroblasts, or myofibroblasts, are thought to be the major source of extracellular matrix production that leads to interstitial fibrosis. To measure myofibroblast accumulation, the marker $\alpha$-SMA was quantified by Western blotting and morphometry. As shown in numerous previous studies, $\alpha$-SMA immunoreactivity was seen in the interstitium after UUO (fig. 6a), and increased $\alpha$ SMA protein expression was detected by Western blotting (fig. 6b). The $\alpha$-SMA-positive tubulointerstitial area was increased significantly after UUO, and was significantly higher in $\mathrm{VE}+/-$ mice than in WT mice at day 7 after UUO (fig. 6a). $\alpha$-SMA protein levels were also sig- 

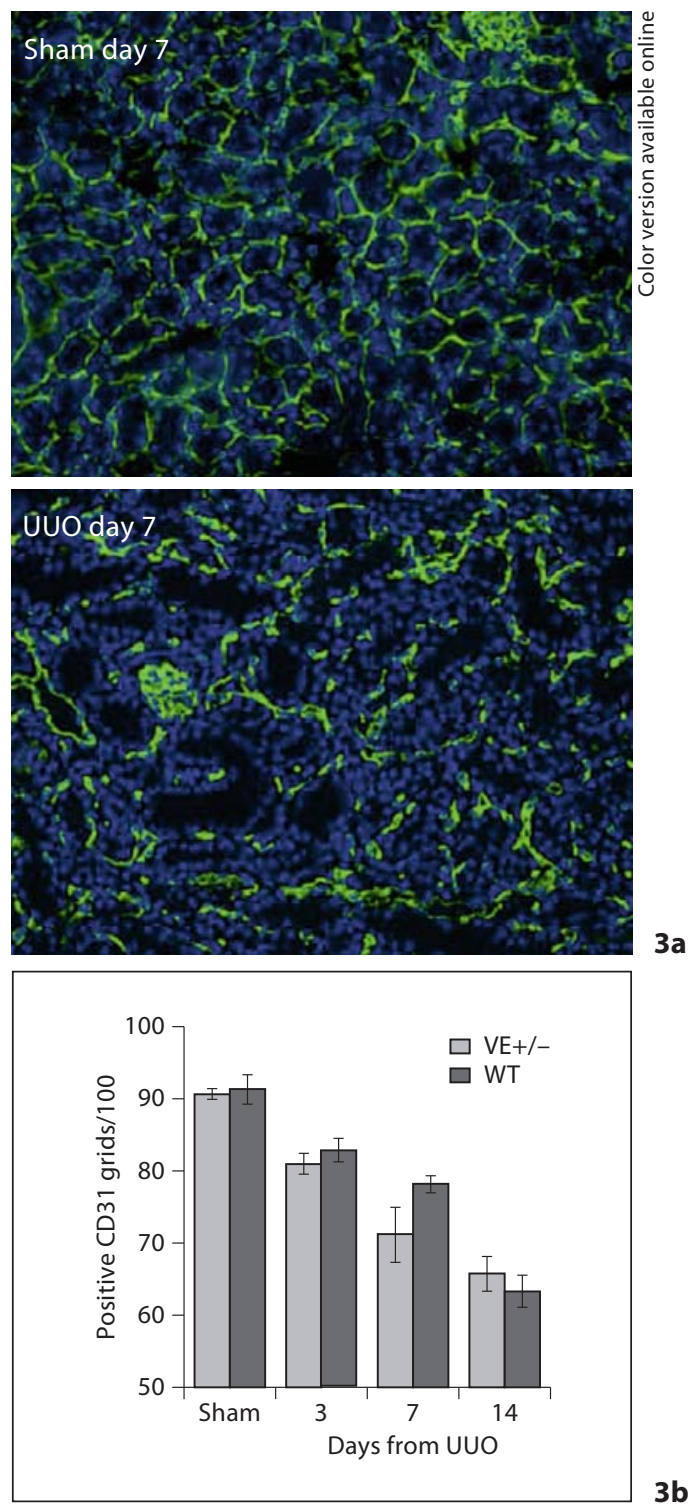

Fig. 3. Peritubular capillary rarefaction. a. Representative CD31 immunostaining of renal tissue from WT mice 7 days after sham surgery (top) and UUO (bottom). $\times 200$. b. Change in peritubular capillary density quantified by grid analysis $(n=4-8)$.

Fig. 4. Vascular permeability after UUO. a Permeability study performed by injection of fluorescent-labeled dextran $(500 \mathrm{kDa}) 7$ days after surgery. Top, WT sham; bottom, WT UUO. $\times 400$. b Permeability study performed by injection of Evans blue dye. Obstructed left kidney (7 days after UUO) in WT mice shows Evans blue accumulation after saline washout, whereas the unobstructed right kidney retained less dye. c Concentration of retained Evans blue at day 7 after UUO. The obstructed kidneys retained much more dye than the contralateral kidneys, showing that vascular permeability was greater. The asterisk indicates significantly greater Evans blue concentration in $\mathrm{VE}+/$ - mice compared to WT $(\mathrm{p}<0.05, \mathrm{n}=6)$.
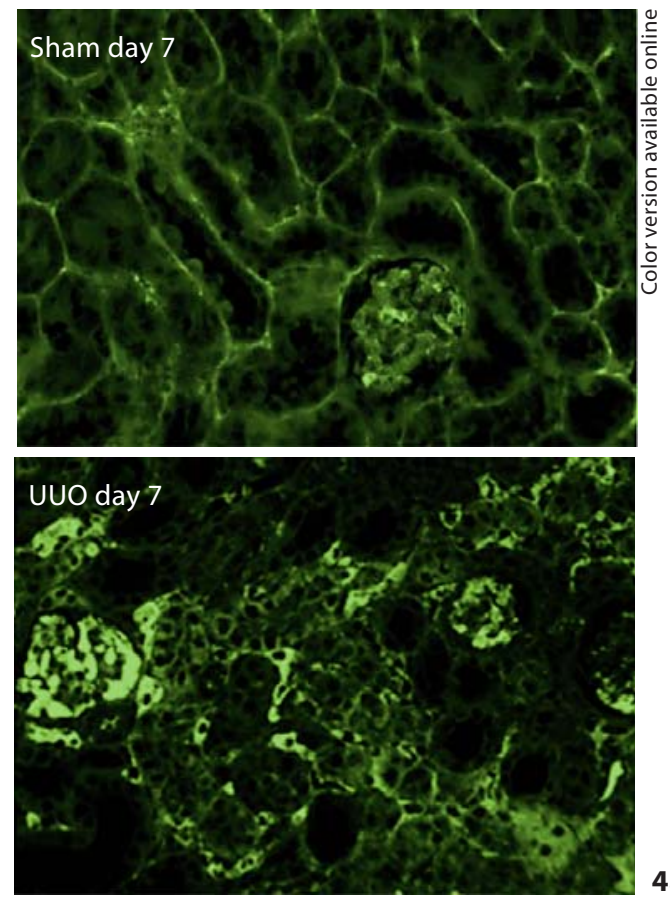

$4 a$
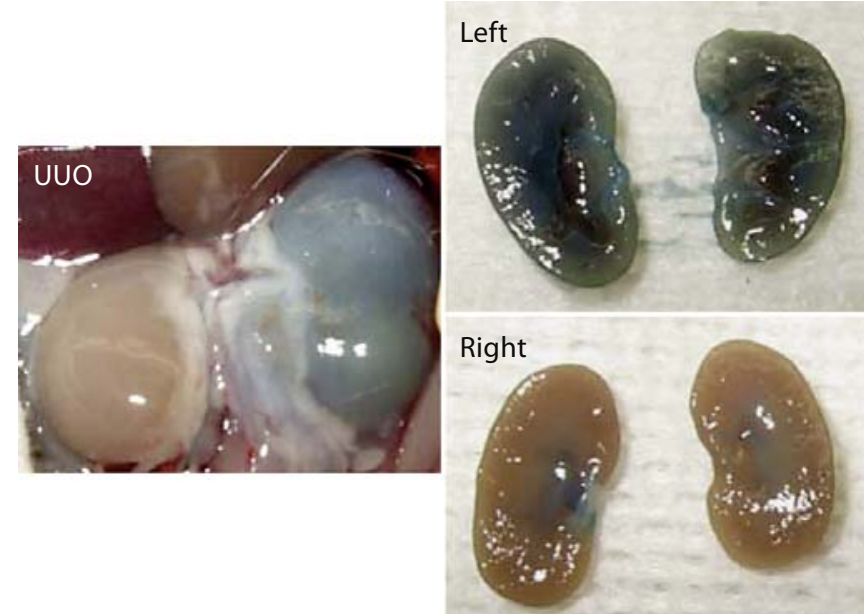

4b

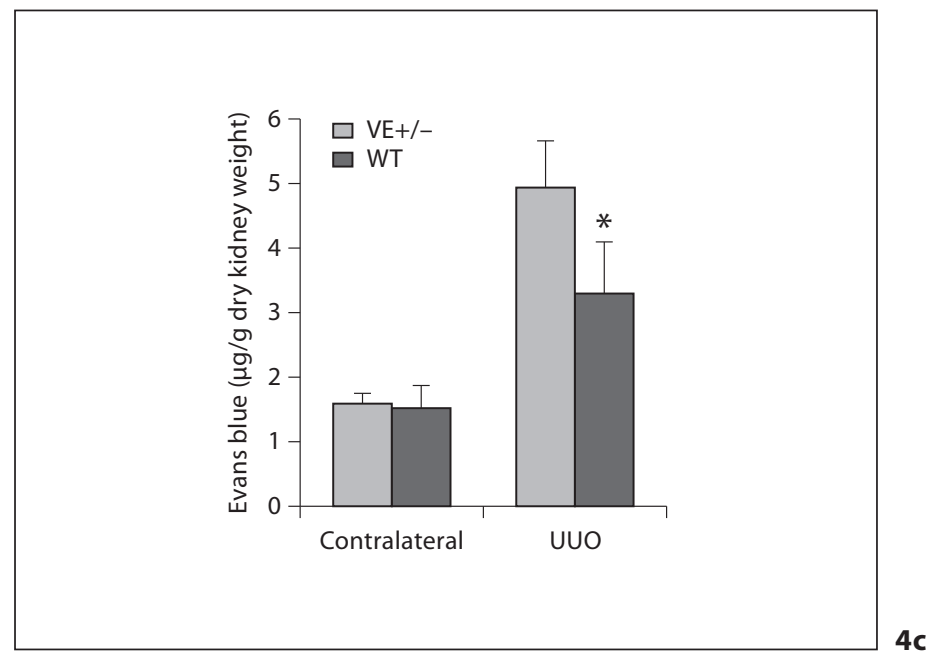


nificantly higher in $\mathrm{VE}+/$ - mice than in WT mice at days 3 and 7 after UUO (fig. 6b), suggesting that myofibroblast accumulation was greater in $\mathrm{VE}+/-$ mice.

Upregulation of Extracellular Matrix Gene Expression Collagen I and III and fibronectin are major extracellular matrix molecules that accumulate in the interstitium during renal fibrosis. In order to investigate how the level of VE-cadherin affects matrix protein expression, the levels of pro-collagen I, pro-collagen III, and fibronectin mRNA were quantified by qRT-PCR. The results show that $\mathrm{VE}+$ /- mice had significantly higher collagen I and III mRNA levels at day 7, but not at day 14 after UUO. Fibronectin transcripts showed a tendency toward higher levels in VE+/- mice than in WT mice at days 7 and 14 after UUO, but these differences were not significant (fig. 7).

The accumulation of interstitial collagen reflects the net effect of differences in collagen synthesis and degradation. Total kidney collagen levels were calculated from the hydroxyproline concentration in hydrolyzed protein extracts from sham and UUO kidneys. Total collagen was significantly increased at days 7 and 14 after UUO compared to sham surgery in both VE+/- and WT mice; however, there were no significant differences between $\mathrm{VE}+/$ and WT (data not shown). Differences were also absent 7 days after UUO when interstitial collagen-positive areas were measured by collagen III immunohistochemistry (data not shown). The discrepancy between collagen gene expression and total collagen may be due to the narrow time window (days 3-7) when VE-cadherin expression differs between VE+/- and WT mice after UUO.

\section{Studies of NG2+ Pericyte Expansion}

Recent studies have suggested that pericytes associated with capillary endothelial cells are precursors of myofibroblasts $[18,19]$. Thus, we hypothesized that a greater expansion of the pericyte population contributed to the increased myofibroblast accumulation seen in $\mathrm{VE}+/-$ mice. To test this hypothesis, we measured the expression of the chondroitin sulfate proteoglycan NG2, which is thought to identify a subset of activated pericytes during development and in pathological conditions.

The distribution of NG2+ pericytes at day 7 after UUO was investigated by double immunofluorescence staining for CD31 and NG2 (fig. 8a). In the sham-operated kidney, NG2 was restricted to a few glomerular mesangial cells and larger vessels. NG2 staining in the tubulointerstitial area was markedly increased at days 3, 7, and 14 after UUO, consistent with an expansion of the pericyte popu-

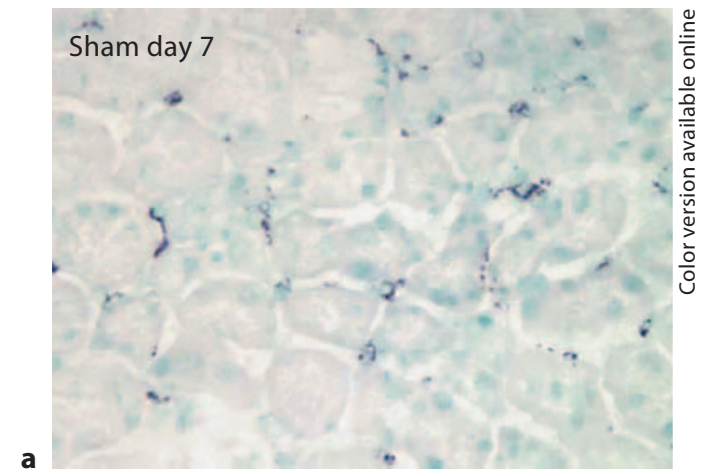

a
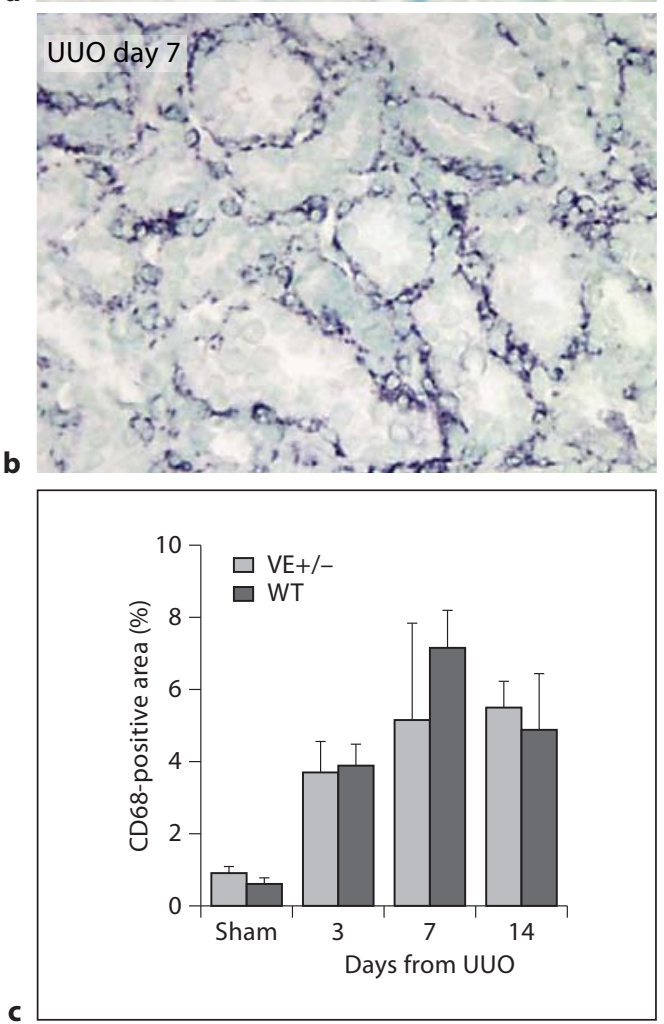

Fig. 5. Expression of the macrophage marker CD68. a CD68 immunohistochemistry in frozen kidney sections prepared 7 days after sham surgery (top) or UUO (bottom) in WT mice. $\times 400$. b The percent positive area was measured in VE+/- and WT mice after sham surgery and 3, 7, and 14 days after UUO. CD68 expression in the tubulointerstitial area was significantly increased relative to sham at days 3,7 , and 14 . However, there was no significant difference between VE+/- and WT mice. ${ }^{*} \mathrm{p}<0.05(\mathrm{n}=5-6)$.

lation. To quantify the total level of NG2 expression, NG2 protein levels were measured by Western blotting (fig. $8 \mathrm{~b}$ ). The level of NG2 was significantly increased at days 3, 7, and 14 after UUO, peaking at day 7. However, contrary to our hypothesis, VE+/- mice showed less NG2 expression than WT mice at day 7 and no significant difference at 


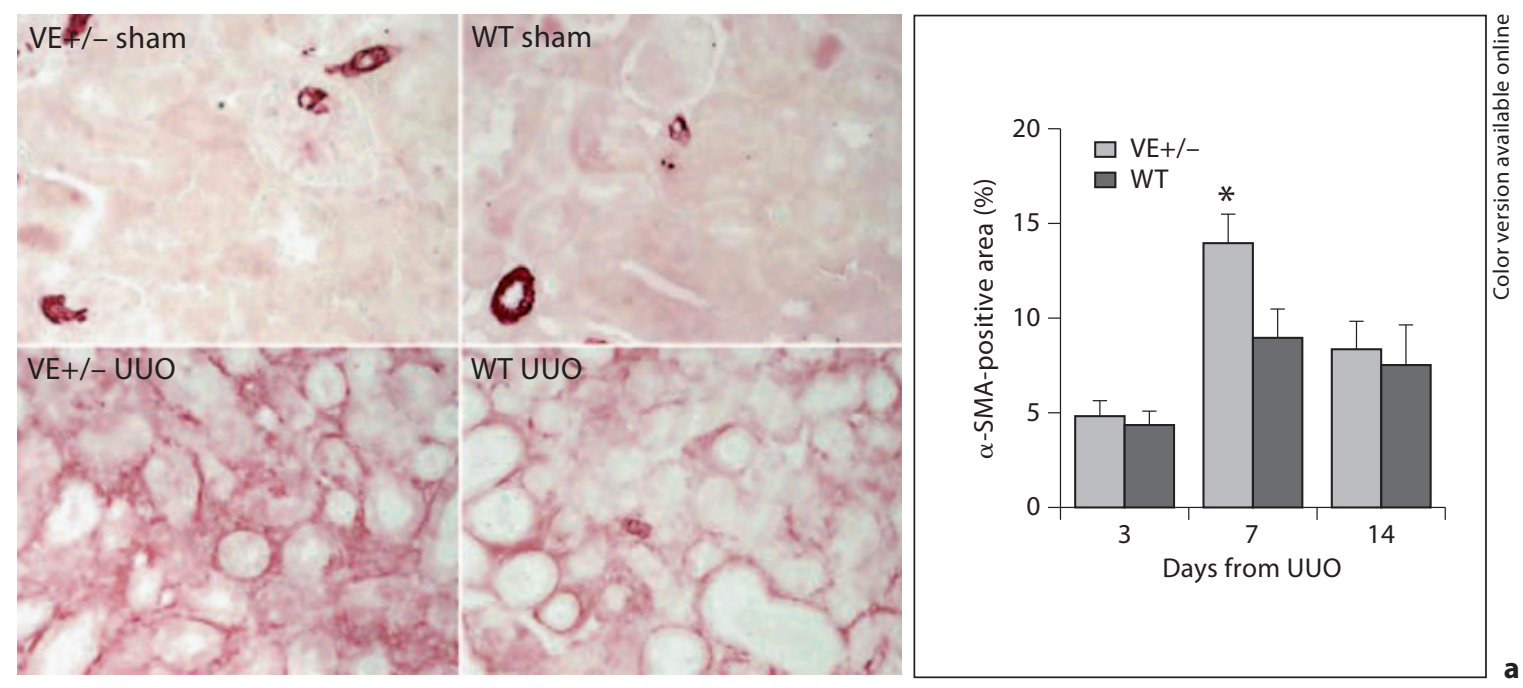

Fig. 6. Expression of the myofibroblast marker $\alpha$-SMA. a Left, immunohistochemistry for $\alpha$-SMA in frozen kidney sections prepared from $\mathrm{VE}+/-$ and WT mice 7 days after sham surgery or UUO. $\times 400$. Right, percent of tubulointerstitial area positive for $\alpha$-SMA was measured. $\alpha$ SMA expression was significantly higher in $\mathrm{VE}+/-$ mice than in $\mathrm{WT}$ at day 7 after UUO. * $\mathrm{p}<0.05$ ( $\mathrm{n}=5-8)$. b Representative Western blot bands for $\alpha$-SMA (quantified using a secondary antibody with an infrared fluorophore) and $\beta$-actin (quantified using an FITC-labeled primary antibody) from $\mathrm{VE}+/$ - and WT mice after sham surgery or UUO. $\alpha$-SMA expression was significantly higher in $\mathrm{VE}+/-$ than WT mice at days 3 and 7 after UUO. ${ }^{*} \mathrm{p}<$ $0.05(\mathrm{n}=5-7)$.
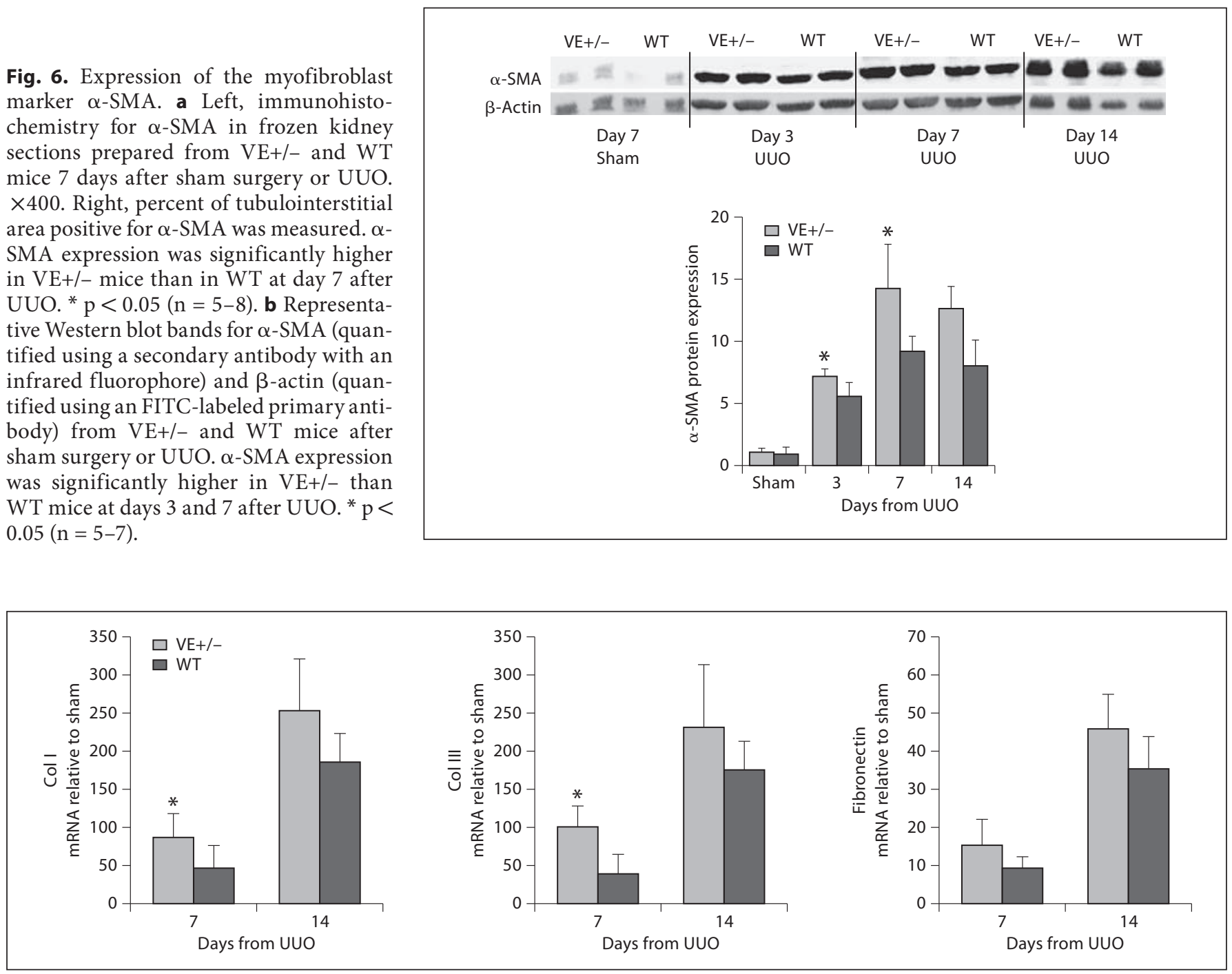

Fig. 7. Relative expression of collagen I, collagen III, and fibronectin mRNA 7 days and 14 days after sham surgery and UUO measured by qRT-PCR and normalized by GAPDH. All values are expressed relative to the mean sham. ${ }^{*} \mathrm{p}<0.05(\mathrm{n}=4-8)$. 

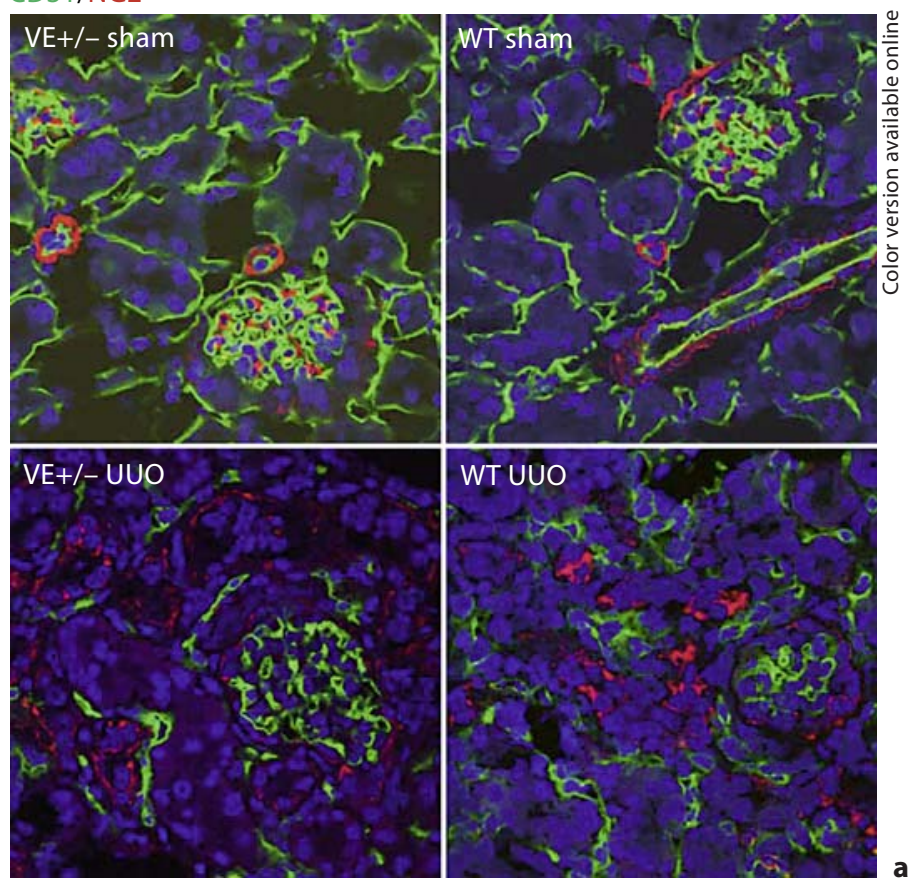

Fig. 8. Expression of the pericyte marker NG2. a Immunostaining of CD31 (green) and NG2 (red) in frozen kidney sections prepared 7 days after sham surgery or UUO from $\mathrm{VE}+/-$ and WT mice. $\times 600$. b Top, representative Western blot bands for NG2 and $\beta$-actin from $\mathrm{VE}+/-$ and $\mathrm{WT}$ mice after sham surgery and UUO. Bar graph compares NG2 protein expression relative to mean sham in $\mathrm{VE}+/-$ and $\mathrm{WT}$ mice at days 3, 7, and 14 after UUO. VE+/mice had significantly lower NG2 expression than WT mice at day 7 after UUO. ${ }^{*} \mathrm{p}<0.05(\mathrm{n}=4-7)$.

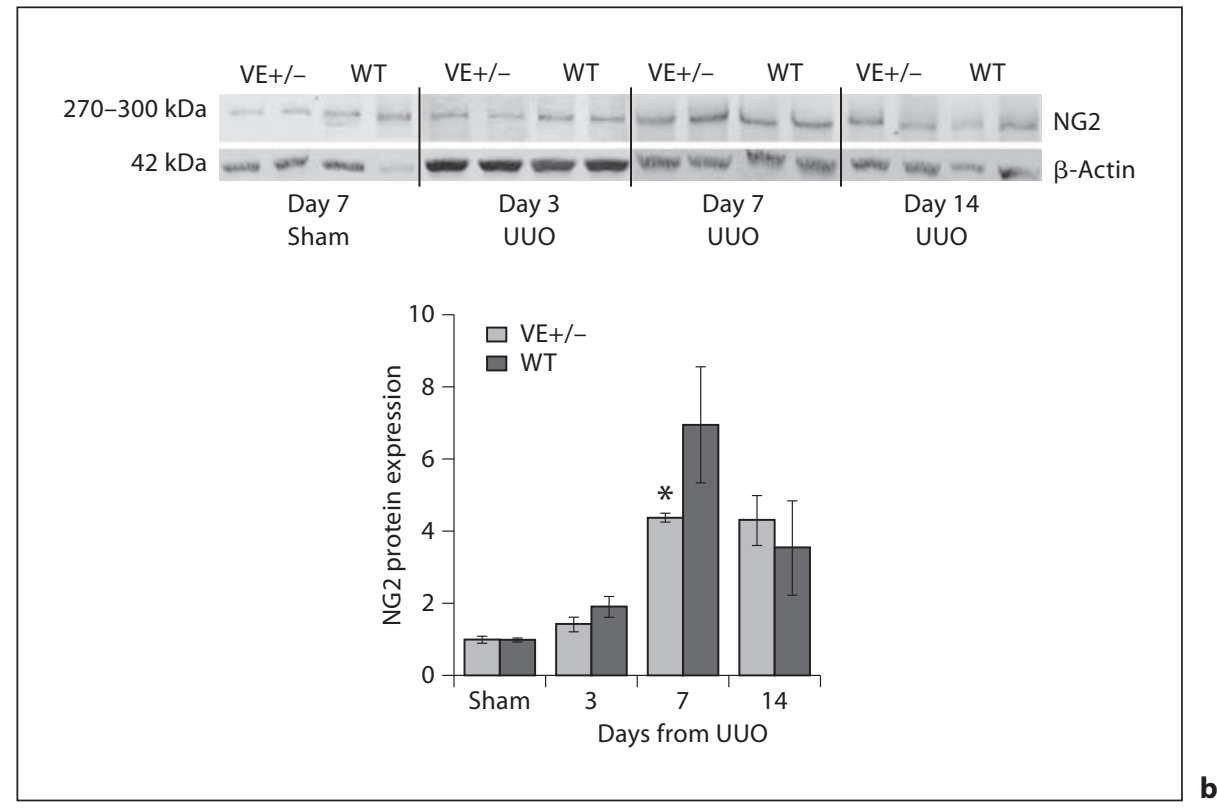

days 3 and 14. These data suggest that the greater myofibroblast accumulation in $\mathrm{VE}+/$ - mice may not be associated with an expansion of the NG2+ pericyte population. However, further studies are required to better understand the roles of pericytes in the UUO model of CKD, and the possible effects of VE-cadherin and other endothelial cell molecules on pericyte differentiation and interstitial migration.

\section{Discussion}

The results of this study demonstrate that peritubular capillaries undergo a significant, progressive rarefaction and show increased permeability in the mouse UUO model of CKD. Unexpectedly, these changes were associated with a significant increase in VE-cadherin protein expression. Based on our immunostaining data and pre- 
vious reports showing that VE-cadherin expression is relatively selective for endothelial cells once embryonic development is complete, VE-cadherin expression after UUO appears to be restricted to the endothelium. Given published evidence that VE-cadherin normally functions to maintain vascular integrity and limit permeability, the current data suggest that VE-cadherin upregulation in endothelial cells represents a compensatory response that serves to reduce the permeability associated with renal damage.

To test this hypothesis, studies using the UUO model of CKD compared outcomes between $\mathrm{VE}+/$ - and WT mice. We found that $\mathrm{VE}+/-$ and WT mice had similar VE-cadherin expression at baseline, in sham-operated kidneys. However, $\mathrm{VE}+/-$ mice showed a delayed increase in VE-cadherin expression from 3 to 7 days after UUO. This short time window likely limited our ability to detect longer-term effects of VE-cadherin deficiency that might have developed if the expression of this gene were suppressed more completely or for a longer period of time. However, we observed several differences in fibrogenic responses between $\mathrm{VE}+/-$ and WT mice. In particular, the kidneys of $\mathrm{VE}+/$ - mice showed a greater increase in vascular permeability, higher levels of the myofibroblast marker $\alpha$-SMA, and higher kidney collagen I and III mRNA levels. These findings are consistent with the hypothesis that the upregulation of VE-cadherin is protective in $\mathrm{CKD}$.

\section{Changes in VE-Cadherin Expression in Renal}

\section{Diseases}

Data on the role of VE-cadherin in renal disease have been limited to studies of acute kidney injury and acute allograft rejection. In an acute kidney injury model (ischemia-reperfusion of bilateral renal arteries in mice and rats), Sutton et al. [33] and Horbelt et al. [37] demonstrated increased renal microvascular permeability together with changes in morphology of the renal microvascular endothelium. These investigators also reported a loss of endothelial cell integrity, alterations in the actin cytoskeleton, and loss of VE-cadherin expression by endothelial cells [33]. VE-cadherin expression was also reduced in acute rejection after renal transplantation, and the downregulation of VE-cadherin was thought to contribute to the acute rejection of allografts [38]. These observations suggest that the mechanisms of capillary injury may differ in acute and chronic renal disease.

In order to determine why VE-cadherin is upregulated after UUO, it will be necessary to better understand the signaling pathways that regulate VE-cadherin transcrip- tion and translation. Several transcription factors have been shown to induce VE-cadherin gene expression, including Ets family members such as Ets1 [39], Erg [40], and TAL-1/SCL [41]. Twist, Snail, and Slug, which are known to regulate epithelial-mesenchymal transition, may also modulate the activity of the VE-cadherin promoter [42]. Some of these transcription factors are regulated by inflammatory and angiogenic signaling molecules released during CKD. For example, Ets-1 can be stimulated by tumor necrosis factor- $\alpha$, fibroblast growth factor- 2 , TGF- $\beta$, and VEGF [43]. Future studies are needed to understand how differences in extracellular signals and transcription factor activation influence the up- or downregulation of VE-cadherin expression in specific disease models.

\section{Implications for the Endothelial Role in Fibrogenesis}

Our data indicate that reduced VE-cadherin expression in heterozygote mice, compared to WT, was associated with more myofibroblast accumulation and higher levels of collagen gene expression. These changes may represent an adaptive response to increased vascular permeability and/or other endothelial cell signals that are triggered in response to renal damage. Our data appear to exclude two possible cellular mechanisms that could connect permeability changes with fibrogenesis. First, we found that macrophage accumulation was not altered in $\mathrm{VE}+/-$ mice. Second, our data suggest that the population of NG2+ pericytes was not increased in $\mathrm{VE}+/-$ mice, relative to WT; in fact, they were significantly lower on day 7. These data are of interest because recent cell lineage tracing studies support the view that pericytes are the major precursors of renal interstitial myofibroblasts in chronically injured kidneys $[18,19]$. In this case, a possible explanation for the findings in the present study is that in $\mathrm{VE}+/$ - mice, NG2+ pericytes differentiated more rapidly into myofibroblasts, under the influence of factors derived from plasma transudate and/ or hyperactivated endothelial cells. This scenario could account for lower expression of NG2 as well as greater myofibroblast accumulation on day 7. A more aggressive pace of pericyte loss in $\mathrm{VE}+/$ - mice might also contribute to greater vascular permeability, as previous studies have shown that pericytes act to maintain vascular structural integrity and induce endothelial cell quiescence $[44,45]$. An alternative possibility is that the pericytes are a heterogeneous population, with only a subset re-expressing NG2 as they transition to myofibroblasts. The possibility that these subpopulations are differently regulated by VE-cadherin deserves further consideration. 
While much remains unknown about the endothelial changes in CKD, our findings suggest that changes in endothelial cell-cell interactions might play a role in fibrogenesis. Further studies are needed to better understand how capillary hyperpermeability, profibrotic signaling interactions, and ultimately capillary loss regulate the severity and reversibility of CKD.

\section{Acknowledgements}

The authors acknowledge research grant support from the National Institutes of Health: DK080926 and HD043376 to I.Y. and DK54500 and DK044757 to A.A.E.; the American Society of Nephrology: Carl W. Gottschalk Research Scholar Grant to I.Y.; Leducq Foundation to C.G. The authors also acknowledge Dr. Elisabetta Dejana (The FIRC Institute of Molecular Oncology, Milan, Italy) for providing VE-cadherin+/- mice and invaluable comments.

\section{Disclosure Statement}

\section{References}

1 Collins AJ, Foley RN, Herzog C, Chavers BM, Gilbertson D, Ishani A, Kasiske BL, Liu J, Mau LW, McBean M, Murray A, St Peter W, Guo H, Li Q, Li S, Peng Y, Qiu Y, Roberts T, Skeans M, Snyder J, Solid C, Wang C, Weinhandl E, Zaun D, Arko C, Chen SC, Dalleska F, Daniels F, Dunning S, Ebben J, Frazier E, Hanzlik C, Johnson R, Sheets D, Wang X, Forrest B, Constantini E, Everson S, Eggers PW, Agodoa L: Excerpts from the US Renal Data System 2009 Annual Data Report. Am J Kidney Dis 2010;55:S1-S420, A426-A427.

-2 Hallan SI, Coresh J, Astor BC, Asberg A, Powe NR, Romundstad S, Hallan HA, Lydersen S, Holmen J: International comparison of the relationship of chronic kidney disease prevalence and ESRD risk. J Am Soc Nephrol 2006; 17:2275-2284.

- 3 Wasse H, McClellan W: Risk for progression to ESRD: further evidence from populationbased studies. J Am Soc Nephrol 2006;17: 2092-2093.

$\checkmark 4$ Eddy AA: Molecular basis of renal fibrosis. Pediatr Nephrol 2000;15:290-301.

$\checkmark 5$ Chevalier RL, Forbes MS, Thornhill BA: Ureteral obstruction as a model of renal interstitial fibrosis and obstructive nephropathy. Kidney Int 2009;75:1145-1152.

6 Boor P, Ostendorf T, Floege J: Renal fibrosis: novel insights into mechanisms and therapeutic targets. Nat Rev Nephrol 2010;6:643656.

7 Kang DH, Hughes J, Mazzali M, Schreiner GF, Johnson RJ: Impaired angiogenesis in the remnant kidney model. II. Vascular endothelial growth factor administration reduces renal fibrosis and stabilizes renal function. J Am Soc Nephrol 2001;12:1448-1457.

-8 Kang DH, Joly AH, Oh SW, Hugo C, Kerjaschki D, Gordon KL, Mazzali M, Jefferson JA, Hughes J, Madsen KM, Schreiner GF, Johnson RJ: Impaired angiogenesis in the remnant kidney model. I. Potential role of vascular endothelial growth factor and thrombospondin-1. J Am Soc Nephrol 2001; 12:1434-1447.

-9 Zhang B, Liang X, Shi W, Ye Z, He C, Hu X, Liu S: Role of impaired peritubular capillary and hypoxia in progressive interstitial fibrosis after 56 subtotal nephrectomy of rats. Nephrology (Carlton) 2005; 10:351-357.

10 Ohashi R, Shimizu A, Masuda Y, Kitamura H, Ishizaki M, Sugisaki Y, Yamanaka N: Peritubular capillary regression during the progression of experimental obstructive nephropathy. J Am Soc Nephrol 2002;13:17951805.

11 Rouschop KM, Claessen N, Pals ST, Weening JJ, Florquin S: CD44 disruption prevents degeneration of the capillary network in obstructive nephropathy via reduction of TGFbeta1-induced apoptosis. J Am Soc Nephrol 2006;17:746-753.

12 Ishii Y, Sawada T, Kubota K, Fuchinoue S, Teraoka S, Shimizu A: Injury and progressive loss of peritubular capillaries in the development of chronic allograft nephropathy. Kidney Int 2005;67:321-332.

13 Choi YJ, Chakraborty S, Nguyen V, Nguyen C, Kim BK, Shim SI, Suki WN, Truong LD: Peritubular capillary loss is associated with chronic tubulointerstitial injury in human kidney: altered expression of vascular endothelial growth factor. Hum Pathol 2000;31: 1491-1497.

14 Seron D, Alexopoulos E, Raftery MJ, Hartley B, Cameron JS: Number of interstitial capillary cross-sections assessed by monoclonal antibodies: relation to interstitial damage. Nephrol Dial Transplant 1990;5:889-893.

15 Manotham K, Tanaka T, Matsumoto M, Ohse T, Miyata T, Inagi R, Kurokawa K, Fujita T, Nangaku M: Evidence of tubular hypoxia in the early phase in the remnant kidney model. J Am Soc Nephrol 2004;15:1277-1288.

16 Matsumoto M, Tanaka T, Yamamoto T, Noiri E, Miyata T, Inagi R, Fujita T, Nangaku M: Hypoperfusion of peritubular capillaries induces chronic hypoxia before progression of tubulointerstitial injury in a progressive model of rat glomerulonephritis. J Am Soc Nephrol 2004; 15:1574-1581.

17 Vestweber D, Winderlich M, Cagna G, Nottebaum AF: Cell adhesion dynamics at endothelial junctions: VE-cadherin as a major player. Trends Cell Biol 2009;19:8-15.

18 Lin SL, Kisseleva T, Brenner DA, Duffield JS: Pericytes and perivascular fibroblasts are the primary source of collagen-producing cells in obstructive fibrosis of the kidney. Am J Pathol 2008; 173:1617-1627.

$\checkmark 19$ Humphreys BD, Lin SL, Kobayashi A, Hudson TE, Nowlin BT, Bonventre JV, Valerius MT, McMahon AP, Duffield JS: Fate tracing reveals the pericyte and not epithelial origin of myofibroblasts in kidney fibrosis. Am J Pathol 2010;176:85-97.

-20 Zeisberg EM, Potenta SE, Sugimoto H, Zeisberg M, Kalluri R: Fibroblasts in kidney fibrosis emerge via endothelial-to-mesenchymal transition. J Am Soc Nephrol 2008; 19: 2282-2287.

21 Dejana E: Endothelial cell-cell junctions: happy together. Nat Rev Mol Cell Biol 2004; 5:261-270.

22 Dejana E, Bazzoni G, Lampugnani MG: Vascular endothelial (VE)-cadherin: only an intercellular glue? Exp Cell Res 1999;252:13-19.

23 Dejana E, Tournier-Lasserve E, Weinstein BM: The control of vascular integrity by endothelial cell junctions: molecular basis and pathological implications. Dev Cell 2009;16: 209-221.

24 Gavard J: Breaking the VE-cadherin bonds. FEBS Lett 2009;583:1-6.

25 Wallez Y, Vilgrain I, Huber P: Angiogenesis: the VE-cadherin switch. Trends Cardiovasc Med 2006;16:55-59.

26 Gulino D, Delachanal E, Concord E, Genoux Y, Morand B, Valiron MO, Sulpice E, Scaife $\mathrm{R}$, Alemany $\mathrm{M}$, Vernet $\mathrm{T}$ : Alteration of endothelial cell monolayer integrity triggers resynthesis of vascular endothelium cadherin. J Biol Chem 1998;273:29786-29793. 
-27 Matsuo S, Lopez-Guisa JM, Cai X, Okamura DM, Alpers CE, Bumgarner RE, Peters MA, Zhang G, Eddy AA: Multifunctionality of PAI-1 in fibrogenesis: evidence from obstructive nephropathy in PAI-1-overexpressing mice. Kidney Int 2005;67:2221-2238.

-28 Yamaguchi I, Lopez-Guisa JM, Cai X, Collins SJ, Okamura DM, Eddy AA: Endogenous urokinase lacks antifibrotic activity during progressive renal injury. Am J Physiol Renal Physiol 2007;293:F12-F19.

-29 Zhang G, Kim H, Cai X, Lopez-Guisa JM, Alpers CE, Liu Y, Carmeliet P, Eddy AA: Urokinase receptor deficiency accelerates renal fibrosis in obstructive nephropathy. J Am Soc Nephrol 2003;14:1254-1271.

>30 Zhang G, Kim H, Cai X, Lopez-Guisa JM, Carmeliet P, Eddy AA: Urokinase receptor modulates cellular and angiogenic responses in obstructive nephropathy. J Am Soc Nephrol 2003;14:1234-1253.

-31 Gerber HP, Hillan KJ, Ryan AM, Kowalski J, Keller GA, Rangell L, Wright BD, Radtke F, Aguet M, Ferrara N: VEGF is required for growth and survival in neonatal mice. Development 1999;126:1149-1159.

>32 Kang DH, Anderson S, Kim YG, Mazzalli M, Suga S, Jefferson JA, Gordon KL, Oyama TT, Hughes J, Hugo C, Kerjaschki D, Schreiner GF, Johnson RJ: Impaired angiogenesis in the aging kidney: vascular endothelial growth factor and thrombospondin-1 in renal disease. Am J Kidney Dis 2001;37:601611.
3 Sutton TA, Mang HE, Campos SB, Sandoval RM, Yoder MC, Molitoris BA: Injury of the renal microvascular endothelium alters barrier function after ischemia. Am J Physiol Renal Physiol 2003;285:F191-F198.

34 Schumacher J, Puchakayala MR, Binkowski K, Eichler W, Dendorfer A, Klotz KF: Effects of candesartan and enalaprilat on the organspecific microvascular permeability during haemorrhagic shock in rats. Br J Anaesth 2006;96:437-443.

35 Rogers DF, Boschetto P, Barnes PJ: Plasma exudation. Correlation between Evans blue dye and radiolabeled albumin in guinea pig airways in vivo. J Pharmacol Methods 1989 21:309-315.

36 Ricardo SD, van Goor H, Eddy AA: Macrophage diversity in renal injury and repair. J Clin Invest 2008; 118:3522-3530.

37 Horbelt M, Lee SY, Mang HE, Knipe NL, Sado Y, Kribben A, Sutton TA: Acute and chronic microvascular alterations in a mouse model of ischemic acute kidney injury. Am J Physiol Renal Physiol 2007;293:F688-F695.

38 Roussoulieres A, McGregor B, Chalabreysse L, Cerutti C, Garnier JL, Boissonnat P, Bastien O, Scoazec JY, Thivolet-Bejui F, Sebbag L, J LM: Expression of VE-cadherin in peritubular endothelial cells during acute rejection after human renal transplantation. J Biomed Biotechnol 2007;2007:41705.

39 Lelievre E, Mattot V, Huber P, Vandenbunder B, Soncin F: ETS1 lowers capillary endothelial cell density at confluence and induces the expression of VE-cadherin. Oncogene 2000;19:2438-2446.
40 Birdsey GM, Dryden NH, Amsellem V, Gebhardt F, Sahnan K, Haskard DO, Dejana E, Mason JC, Randi AM: Transcription factor ERG regulates angiogenesis and endothelial apoptosis through VE-cadherin. Blood 2008;111:3498-3506.

41 Deleuze V, Chalhoub E, El-Hajj R, Dohet C, Le Clech M, Couraud PO, Huber P, Mathieu D: TAL-1/SCL and its partners E47 and LMO2 up-regulate VE-cadherin expression in endothelial cells. Mol Cell Biol 2007;27: 2687-2697.

42 Lopez D, Niu G, Huber P, Carter WB: Tumor-induced upregulation of twist, snail, and slug represses the activity of the human VE-cadherin promoter. Arch Biochem Biophys 2009;482:77-82.

43 Wernert N, Raes MB, Lassalle P, Dehouck MP, Gosselin B, Vandenbunder B, Stehelin $\mathrm{D}$ : C-ETS1 proto-oncogene is a transcription factor expressed in endothelial cells during tumor vascularization and other forms of angiogenesis in humans. Am J Pathol 1992; 140:119-127.

44 Diaz-Flores L, Gutierrez R, Madrid JF, Varela H, Valladares F, Acosta E, Martin-Vasallo P, Diaz-Flores L Jr: Pericytes. Morphofunction, interactions and pathology in a quiescent and activated mesenchymal cell niche. Histol Histopathol 2009;24:909-969.

45 von Tell D, Armulik A, Betsholtz C: Pericytes and vascular stability. Exp Cell Res 2006;312: 623-629. 